\title{
Properties of singular vectors using convective available potential energy as final time norm
}

Article

Published Version

Stappers, R. J. J. and Barkmeijer, J. (2011) Properties of singular vectors using convective available potential energy as final time norm. Tellus Series A: Dynamic Meteorology and Oceanography, 63 (3). pp. 373-384. ISSN 0280-6495 doi: https://doi.org/10.1111/j.1600-0870.2010.00501.x Available at https://centaur.reading.ac.uk/28438/

It is advisable to refer to the publisher's version if you intend to cite from the work. See Guidance on citing.

To link to this article DOI: http://dx.doi.org/10.1111/j.1600-0870.2010.00501.x

Publisher: Wiley-Blackwell

All outputs in CentAUR are protected by Intellectual Property Rights law, including copyright law. Copyright and IPR is retained by the creators or other copyright holders. Terms and conditions for use of this material are defined in the End User Agreement.

www.reading.ac.uk/centaur 
Central Archive at the University of Reading

Reading's research outputs online 


\title{
Properties of singular vectors using convective available potential energy as final time norm
}

\author{
By ROEL STAPPERS* and JAN BARKMEIJER Royal Netherlands Meteorological Institute (KNMI), \\ 3732 GK De Bilt, The Netherlands
}

(Manuscript received 25 March 2010; in final form 6 December 2010)

\begin{abstract}
We study the feasibility of using the singular vector technique to create initial condition perturbations for shortrange ensemble prediction systems (SREPS) focussing on predictability of severe local storms and in particular deep convection. For this a new final time semi-norm based on the convective available potential energy (CAPE) is introduced. We compare singular vectors using the CAPE-norm with SVs using the more common total energy (TE) norm for a 2-week summer period in 2007, which includes a case of mesoscale extreme rainfall in the south west of Finland. The CAPE singular vectors perturb the CAPE field by increasing the specific humidity and temperature of the parcel and increase the lapse rate above the parcel in the lower troposphere consistent with physical considerations. The CAPE-SVs are situated in the lower troposphere. This in contrast to TE-SVs with short optimization times which predominantly remain in the high troposphere. By examining the time evolution of the CAPE singular values we observe that the convective event in the south west of Finland is clearly associated with high CAPE singular values.
\end{abstract}

\section{Introduction}

Due to the success of ensemble prediction systems (EPSs) for the medium range several numerical weather prediction consortia have developed, or are in the process of developing, EPSs for the short range (SREPS) (see e.g. Hágel and Horányi, 2007; Iversen et al., 2007; Du et al., 2009). The main goal of these short-range ensemble prediction systems is to aid forecasters especially in situations of severe weather. Almost all severe local storms are associated with deep convection and require three necessary ingredients (Doswell, 1987; Johns and Doswell, 1992; Schultz and Schumacher, 1999).

(i) A moist layer of sufficient depth

(ii) A steep enough lapse rate

(iii) Sufficient lifting of a parcel from the moist layer to allow it to reach its level of free convection (LFC).

The requirement for moisture (i) and steep enough lapse rate (ii) can be understood in terms of convective available potential energy (CAPE). In general the large-scale flow creates the favourable thermodynamic environment while the mesoscale provides the lift needed to initiate convection (Doswell, 1987).

In medium range EPS several methods are being used to create initial condition perturbations. At the European Centre for

\footnotetext{
* Corresponding author. e-mail: stappers@knmi.nl DOI: $10.1111 / \mathrm{j} .1600-0870.2010 .00501 . x$
}

Medium-Range Weather Forecasts (ECMWF) the initial condition perturbations are created by taking linear combination of singular vectors. Singular vectors are norm dependent structures and can therefore be designed to focus on particular forecast aspects. Given the requirement that the SREPS should inform about predictability of severe local storms in general and deep convection in particular, we investigate the properties of singular vectors that have maximum impact on the $12 \mathrm{~h}$ CAPE forecasts. We will refer to these singular vectors as CAPE-SVs.

Based on the fact that forecasters are able to improve numerical weather predictions of mesoscale convective systems, $\mathrm{Xu}$ et al. (2001) propose to let forecasters identify the area of forecast concern and use the adjoint model to determine key atmospheric parameters influencing the forecast. The output from these sensitivity experiments is then used to create initial condition perturbations for a short-range ensemble prediction system, which is specifically designed to investigate the forecast concern of the day. They introduced nine cost functions that are believed to be important for deep convection. In their conclusions they state that with the perturbation sizes they used their method is superior in terms of the equitable threat scores and ranked probability scores between 6 and $24 \mathrm{~h}$ to ensembles based on physics perturbations or a certain Monte Carlo approach both described in Stensrud et al. (2000). Homar et al. (2006) compared an EPS using the method of Xu et al. (2001) to the National Centers for Environmental Prediction (NCEP) operational Short Range Ensemble Forecasting (SREF) system and the Eta model for a 3-week period. They show that the adjoint based system 
improved the numerical forecasts of severe weather and produces better probabilistics scores of heavy precipitation than the SREF. We show that by a suitable projection the CAPE-SVs are equal to optimal perturbations and could therefore be used as a cost function in the method of Xu et al. (2001).

One of the assumptions that has to be made in the SV calculation is that forecast errors are linearly related to initial condition errors. When going to higher resolutions and at the same time focusing on mesoscale aspects of the forecast, such as instantaneous convective precipitation, the linearity assumption becomes increasingly problematic, see Mahfouf and Bilodeau (2007), Hohenegger and Schär. (2007) and Errico et al. (2003). As CAPE is associated with the large-scale flow and the CAPEfield is a relatively smooth field compared to convective precipitation it is plausible that the tangent linear assumption holds for longer lead times compared to convective precipitation.

In Section 2, we introduce singular vectors and derive a norm based on CAPE. We show how the CAPE-norm can be used in adjoint sensitivity studies and for the creation of optimal perturbations. In Section 3, we investigate the properties of the CAPE-SVs for a 2-week summer period in 2007 and compare them with TE-SVs. In Section 4, we discuss the validity of the tangent linear assumption using twin experiments. Finally, in Section 5 we end with conclusions.

\section{Theory}

\subsection{Singular vectors}

In the singular vector calculation it is assumed that forecast errors $\epsilon(T)$ are linearly related to errors $\epsilon(0)$ at analysis time

$\epsilon(T)=\mathbf{M}(T, 0) \epsilon(0)$,

where $\mathbf{M}(T, 0)$ is called the propagator or resolvent. The action of $\mathbf{M}$ on a vector $\epsilon(0)$ is obtained by integrating the tangent linear model

$\dot{\epsilon}(t)=J[x(t)] \epsilon(t)$

starting from the initial condition $\epsilon_{0}$ and where $x(t)$ is the solution of the non-linear forecast model

$\dot{x}=F(x)$

starting from the initialized analysis $x_{0}$. There are practical problems with obtaining the tangent linear model by linearizing the non-linear atmospheric model such that $J[x(t)]=\left.\frac{\partial F}{\partial x}\right|_{x(t)}$ due to the strong non-linearities in the physical parametrizations of the models (Vukićević and Errico, 1993; Janisková, 2003; Fillion and Bélair, 2004).

Singular vectors are vectors $\epsilon(0)$ that maximize the ratio

$$
\frac{[\mathbf{P} \epsilon(T), \mathbf{D P} \epsilon(T)]}{[\epsilon(0), \mathbf{E} \epsilon(0)]},
$$

where $\mathbf{D}$ is a positive semi-definite symmetric operator, $\mathbf{E}$ is a positive definite symmetric operator w.r.t. the Euclidean inner product $(\cdot, \cdot)$ and $\mathbf{P}$ is a projection operator (Barkmeijer et al., 2001). By using Lagrange multipliers it is easy to show that the solution to eq. (4) is equal to the leading eigenvector of the following generalized eigenvector problem.

$\mathbf{M}^{*} \mathbf{P}^{*} \mathbf{D P M} \epsilon(0)=\lambda \mathbf{E} \epsilon(0)$.

Here $\mathbf{M}^{*}$ is the adjoint of the propagator $\mathbf{M}$ w.r.t. the inner product $(\cdot, \cdot)$. This is a symmetric generalized eigenvalue problem and therefore the eigenvalues are real and can be ordered $\lambda_{1} \geq$ $\lambda_{2} \geq \ldots \geq \lambda_{N} \geq 0$. The $n$th singular vector maximizes the ratio in eq. (4) in a direction E-orthogonal to the space spanned by the leading $(n-1)$ singular vectors. For each singular vector the singular value is given by $\sqrt{\lambda}$.

\subsection{CAPE norm}

The convective available potential energy based on the virtual temperature is defined as (Doswell and Rasmussen, 1994)

$\mathrm{CAPE}=g \int_{\mathrm{LFC}}^{\mathrm{EL}} \frac{T_{\mathrm{v}}\left(z^{\prime}\right)-\bar{T}_{\mathrm{v}}\left(z^{\prime}\right)}{\bar{T}_{\mathrm{v}}\left(z^{\prime}\right)} \mathrm{d} z^{\prime}$,

where $T_{\mathrm{v}}$ is the virtual temperature of the parcel rising adiabatically from its source layer and $\bar{T}_{\mathrm{v}}$ is the virtual temperature of the environment. The integral is only evaluated between heights where the integrand is positive. These heights are called the level of free convection (LFC) and the equilibrium level (EL). For computation simplicity we derived the final time CAPE-norm from a simplified CAPE calculation used in the post processing of an earlier version of the operational ECMWF-model (Bechtold, personal communication, 2008)

$\mathrm{CAPE}=g \int_{\mathrm{LFC}}^{\mathrm{EL}} \frac{\theta_{e_{\mathrm{up}}}-\theta_{e_{\mathrm{sat}}}(z)}{\theta_{e_{\mathrm{sat}}}(z)} \mathrm{d} z^{\prime}$,

where $\theta_{e_{\text {up }}}$ is the equivalent potential temperature of a parcel based on the average conditions in the lowest $50 \mathrm{hPa}$ in the model and $\theta_{e_{\text {sat }}}$ is the saturation equivalent potential temperature. The value of $\theta_{e_{\text {up }}}$ is kept constant during the parcel ascent. The CAPE values from eq. (7) are approximately $25 \%$ higher than those computed using eq. (6) (Bechtold, personal communication). Note that multiplying the final time norm in the singular vector calculation by a scalar has no impact on the structure of the singular vector. From the Taylor expansion of the CAPE calculation around a model state $x$ we obtain

$\operatorname{CAPE}(x+\delta x)-\operatorname{CAPE}(x)=\frac{\partial \mathrm{CAPE}}{\partial x} \delta x+O\left(\delta x^{2}\right)$.

Therefore the impact of an infinitesimal perturbation $\delta x$ of the model state $x$ on CAPE, measured in the Euclidean norm, is

$\|\operatorname{CAPE}(x+\delta x)-\operatorname{CAPE}(x)\|^{2}=\|\mathbf{C} \delta x\|^{2}=\left(\delta x, \mathbf{C}^{*} \mathbf{C} \delta x\right)$. 
We will refer to $\mathbf{C}=\frac{\partial \mathrm{CAPE}}{\partial x}$ as the CAPE-operator. This operator maps three-dimensional perturbations of the model state to two-dimensional CAPE perturbations. The operator $\mathbf{C}^{*} \mathbf{C}$ is positive semi-definite and therefore defines a semi-norm on the model states.

The eigenvalue problem (5) can now be written as

$\mathbf{E}^{-1 / 2} \mathbf{M}^{*} \mathbf{P}^{*} \mathbf{C}^{*} \mathbf{C P M E} \mathbf{E}^{-1 / 2} v=\lambda v$

where we have assumed that $\mathbf{E}$ is a simple metric in the sense that $\mathbf{E}^{-1 / 2}$ can easily be obtained, for example, the metric used in the TE-norm (Errico et al., 2004). The solutions to the original maximization problem are now given by $\epsilon=\mathbf{E}^{-1 / 2} v$.

\subsection{Squared mean versus mean squared SVs}

Both positive and negative CAPE perturbations contribute to the CAPE norm. This typically leads to nearby areas of negative and positive CAPE perturbations, similar to the wave train structures when the TE-norm is used. In particular there is the possibility that perturbations which are large in the CAPE-norm have small impact on the mean CAPE value in a certain area. This may prohibit the analysis of how CAPE perturbations are associated with, for example, severe convective events. For this reason we also computed perturbations that have maximum impact on the square of the mean CAPE values as opposed to the mean of squared CAPE values. We will refer to these SVs as SCAPE-SVs. If we define a projection operator $\Sigma$ on the CAPE perturbations as

$\Sigma=\left[\begin{array}{ccc}1 & \ldots & 1 \\ 0 & 0 & 0 \\ \vdots & \vdots & \vdots \\ 0 & 0 & 0\end{array}\right]$

Then, maximizing the squared mean CAPE perturbation using the total energy metric $\mathbf{E}$ at initial time is equivalent to solving the eigenvector problem

$\mathbf{E}^{-1 / 2} \mathbf{M}^{*} \mathbf{P}^{*} \mathbf{C}^{*} \Sigma^{*} \Sigma \mathbf{C P M E}^{-1 / 2} v=\lambda v$.

The projection operator $\Sigma$ is a rank 1 mapping. Therefore there is only one singular vector with $\sigma \neq 0$. This singular vector is obtained up to a normalization by

$\epsilon=\mathbf{E}^{-1} \mathbf{M}^{*} \mathbf{P}^{*} \mathbf{C}^{*} 1$.

Here 1 denotes a vector of ones. In particular, there is no need for a Lanczos scheme to determine this 'leading' SCAPE-SV.

Similar expressions are obtained in Mahfouf and Bilodeau (2007) and Errico et al. (2003), where a cost function is maximized subject to constraints on the initial conditions. The resulting structures are referred to as optimal perturbations. The equivalence between their formulation and the squared mean $\mathrm{SV}$ s given here can be demonstrated by defining a cost-function as the sum of CAPE-values inside a projection domain

$$
J=[\tilde{\mathbf{P C A P E}}(x), 1] .
$$

Here $\tilde{\mathbf{P}}$ is a projection operator that sets CAPE-perturbations outside the projection domain to zero. Small perturbations $\delta x$ in the model state lead to perturbations in CAPE

$\delta \mathrm{CAPE}=\mathbf{C} \delta x$,

where $\mathbf{C}$ is the CAPE operator. We get

$\delta J=(\tilde{\mathbf{P}} \delta \mathrm{CAPE}, 1)=\left(\mathbf{C} \delta x_{T}, \tilde{\mathbf{P}} 1\right)=\left(\delta x_{0}, \mathbf{M}^{*} \mathbf{C}^{*} \tilde{\mathbf{P}} 1\right)$

and by definition

$\delta J=\left(\nabla_{x_{0}} J, \delta x_{0}\right)$.

So we conclude that the adjoint sensitivity of the sum of CAPE is given by

$\nabla_{x_{0}} J=\mathbf{M}^{*} \mathbf{C}^{*} \tilde{\mathbf{P}}^{*} 1$.

Maximization of the cost function under constraints on the initial conditions and introducing a projection $\mathbf{P}$ such that $\tilde{\mathbf{P}} \mathbf{C}=$ CP leads to expression (13). The optimal pertubations maximize the mean CAPE values while SCAPE-SVs maximize the square of the mean CAPE values. Therefore the remark in Mahfouf and Bilodeau (2007) that SVs are not equal to optimal perturbations is true but only in the sense that the SVs do not necessarily increase the mean CAPE values. The structures obtained using both methods are equal. The CAPE cost function can now be used in the method of Xu et al. (2001) to create initial condition perturbation.

\section{Case study}

For all experiments we used HIRLAM 7.3beta1 with incremental 4DVAR using two outer loops and digital filtering initialization (DFI) as a weak constraint. The domain is approximately equal to the Euro-Atlantic region $\left(\approx 40^{\circ} \mathrm{W}-40^{\circ} \mathrm{E} 35^{\circ} \mathrm{N}-80^{\circ} \mathrm{N}\right)$ with a resolution of $0.5^{\circ}$. The singular vectors are calculated every $6 \mathrm{~h}$ with an optimization time of $12 \mathrm{~h}$ for a 2 -week summer period starting from August 15, 2008 at 00 UTC till August 30, 12 UTC. This period is also used in the grand limited area model ensemble prediction system (GLAMEPS) project (Iversen et al., 2007) to test different short-range ensemble strategies. During this 2week period a severe mesoscale convective system developed in the south west of Finland, which the operational Finnish model failed to predict in any cycle verifying at the same time (Iversen et al., 2007). We use analysis blending (Yang, 2005) such that the large-scales are mixed with the ECMWF analysis. For a detailed description of the HIRLAM system we refer to Undén et al. (2002). We use the default settings for the tangent linear model which only includes vertical diffusion of the Météo France simplified physics package (Janisková et al., 1999). The non-linear trajectory was updated every hour in the tangent linear model. At final time we target the fields to the area defined by 


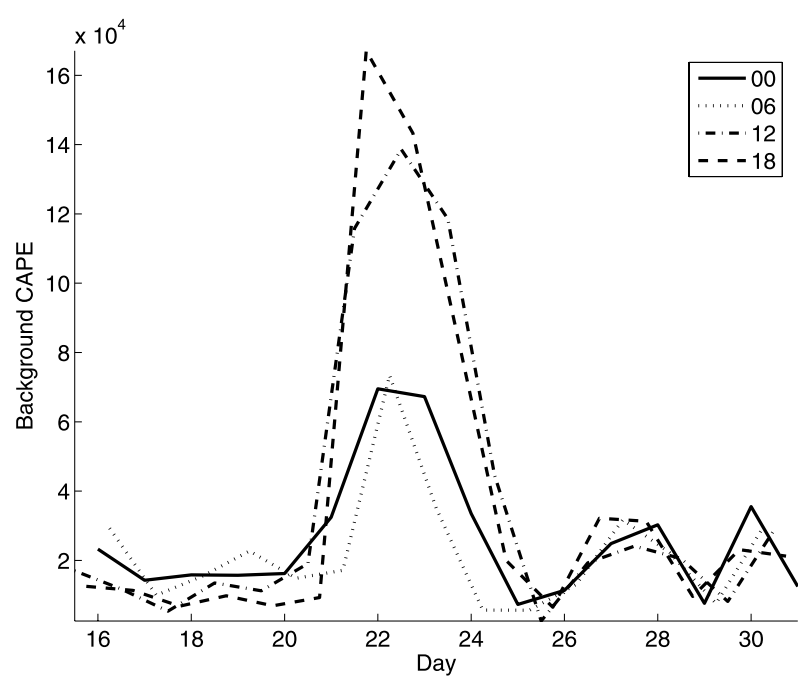

Fig. 1. Sum of CAPE values inside the projection domain for the $12 \mathrm{~h}$ forecast as a function of the verification time.

$55^{\circ} \mathrm{N}-65^{\circ} \mathrm{N}$ and $10^{\circ} \mathrm{E}-30^{\circ} \mathrm{E}$. At initial time we use a dry total energy norm similar to the computation of singular vectors used in the EPS at ECMWF (Leutbecher and Palmer, 2008), that is, we do not perturb the specific humidity field.
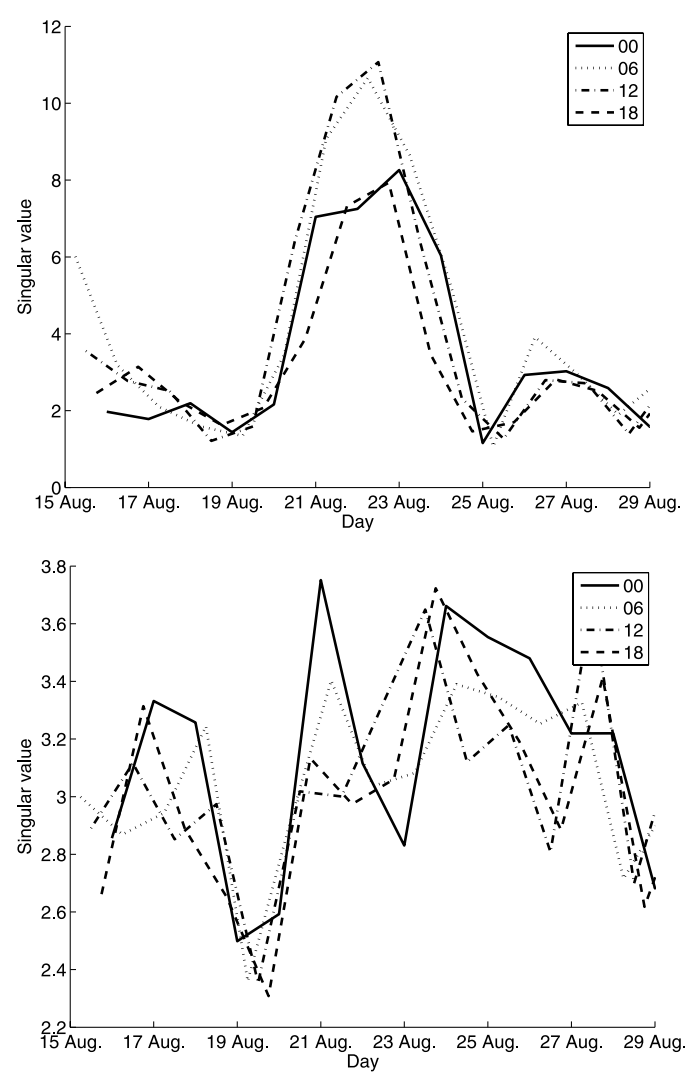

Figure 1 shows the sum of CAPE values inside the projection domain for the $12 \mathrm{~h}$ forecast as a function of verification time using the approximate CAPE calculation from which the CAPE norm was derived. Around August 22, during the mesoscale convective event, the CAPE values are relatively high and during this period there is a clear diurnal cycle with lower CAPE values for forecasts that verify at 00 and 06 UTC, likely due to the cooler, stable boundary layer that develops at night.

\subsection{Singular values}

In all singular value plots we plot the average singular values of the leading ten SVs for the TE-SVs and CAPE-SVs and the singular value for leading SCAPE-SVs. We will refer to these as the average singular value. Figure 2 shows the average singular value using the final time TE-norm, CAPE-norm and SCAPEnorm. Both the CAPE singular values and the SCAPE singular values clearly indicate that the leading singular vectors have a large impact on the CAPE in the background flow around August 22. In the period around August 22 there is a daily cycle in the CAPE singular values with lower singular values for forecasts starting from 00 and 18 UTC.

By comparing the CAPE and SCAPE singular values with Fig. 1 it is clear that the higher singular values around August
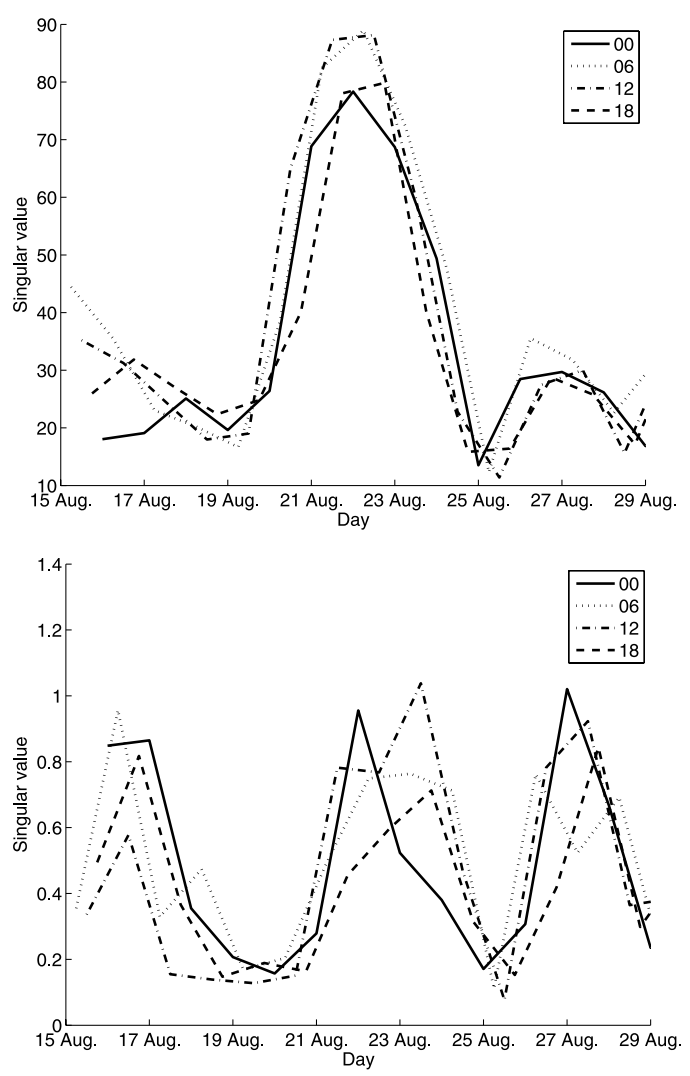

Fig. 2. Average singular value of the CAPE-SV (top left-hand panel), SCAPE-SV (top right-hand panel) and TE-SVs (bottom left-hand panel) as a function of the analysis time. In the lower right-hand panel we show the average 'singular value' of the TE-SVs in the CAPE-norm. 
22 correspond to high CAPE values in the $12 \mathrm{~h}$ forecasts inside the projection domain during this period. Note however that the low CAPE values verifying at 00 UTC correspond to relatively high singular values according to the SCAPE and CAPE singular values starting from the 12 UTC analysis of the previous day. These results should be contrasted with the singular values for the TE-SVs. Here there is no increase in the singular values around August 22 and no daily cycle. In Fig. 2 (lower right-hand panel) we show the impact of the TE-SVs on CAPE, that is, we measure the size of the evolved TE-SVs in the CAPE-norm. In this case we do see a clear signal in the 'singular values'. By comparing with the singular values of the CAPE-SVs we see that the CAPE-SVs are approximately 10 times more effective at perturbing CAPE compared to TE-SVs around August 22.

\subsection{SV structures for August 21 at 18 UTC}

The mesoscale convective system in the south west of Finland developed around August 22 at 6 UTC. Here we discuss the properties of the SVs using the TE, CAPE and SCAPE norm starting from the August 2118 UTC analysis and verifying at August 22 at 6 UTC. Figure 3 shows the structure at initial and final time of the leading TE-SVs. At initial time the temperature perturbations show the typical tilted wave train structure against the wind-shear. After $12 \mathrm{~h}$ the structures are untilted and have propagated upward towards the jet-stream level. These characteristics are well known features of TE-SVs (Palmer et al.,
1998). Due to the short optimization time used here the temperature perturbations at initial time are high in the troposphere. At initial time the energy is almost completely in the temperature field which is converted to kinetic energy at final time. These are consistent features of all the TE-SVs in the 2-week period (see Fig. 6).

Figures 4 and 5 show the structure at initial and final time of the leading CAPE-SV and the leading SCAPE-SV. Compared with the leading TE-SV both types of CAPE-SVs are situated much lower in the troposphere and the CAPE-SV is more localized in the horizontal. At final time the temperature field is increased in the low troposphere and decreased in the mid troposphere for both the CAPE and SCAPE-SVs. The impact of the evolved TE-SVs on CAPE is small compared to the CAPE-SVs.

For this particular day the leading CAPE-SVs increases CAPE for almost all grid points inside the projection domain and therefore also increases the mean CAPE values, that is, we do not have wave train structures in the evolved CAPE-field. As a result of this the SCAPE-SV, which maximizes the mean CAPE, projects strongly on the CAPE-SVs in the southwest of the projection domain. More generally, using the sign ambiguity in the CAPESVs, we can multiply all CAPE-SVs with the sign of the mean evolved CAPE field inside the projection domain such that the CAPE-SVs all have a non-negative impact on the mean CAPEfield. When the SCAPE-SV is written as a linear combination of these CAPE-SVs it will mainly project on those CAPE-SVs that have a large positive bias in the mean CAPE field. Being linear
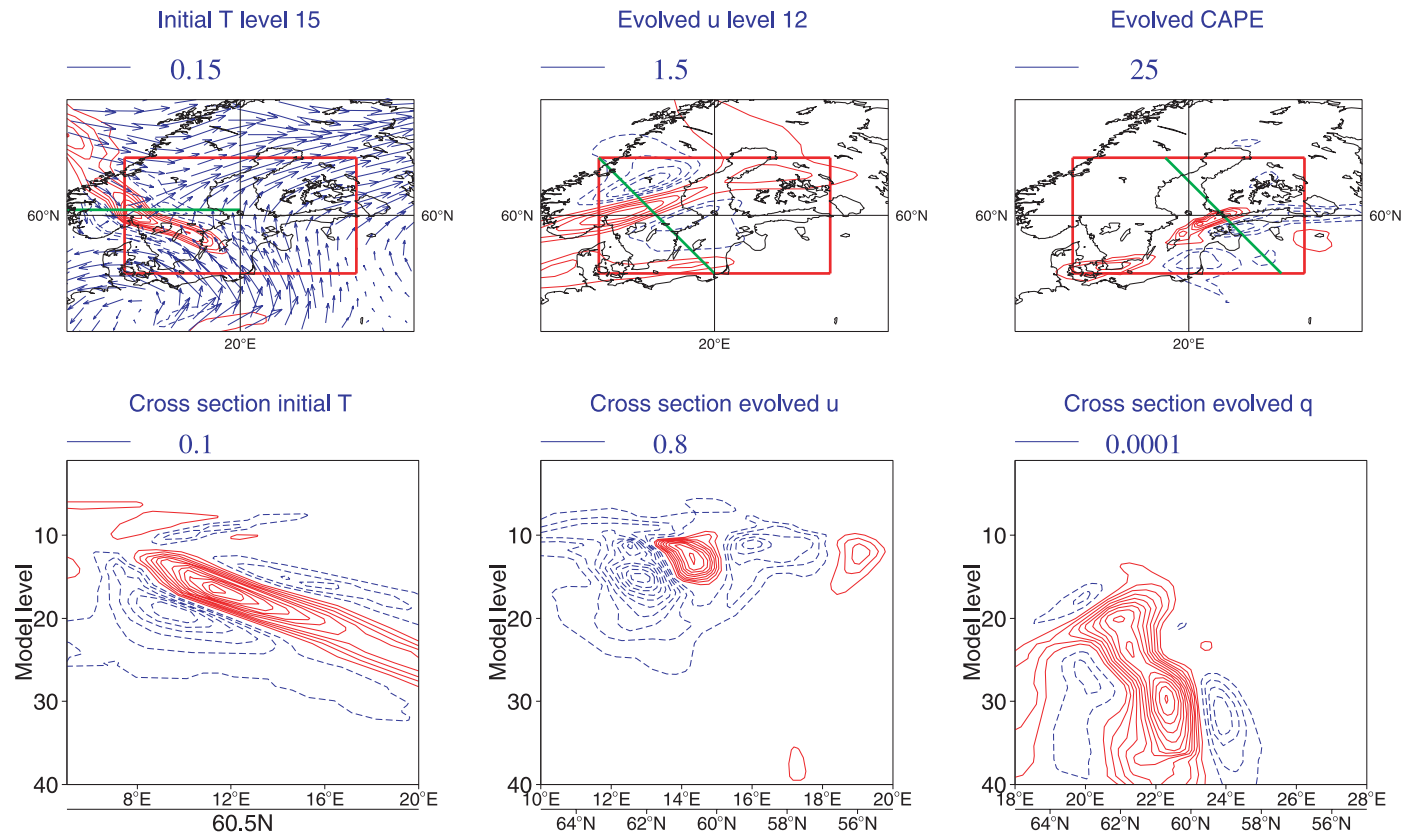

Fig. 3. Leading TE-SVs for August 21 at 18 UTC, initial temperature and background wind at model level 15 (top left-hand panel) cross section initial temperature (bottom left-hand panel), evolved u-component wind at level 12 (top middle panel), cross section u-component wind (bottom middle panel), Evolved CAPE (top right-hand panel) and cross section specific humidity (bottom right-hand panel). The vertical cross sections are along the green line in the upper plot. The red box indicates the target area for the SVs. Positive perturbations (solid red), negative perturbations (dashed blue). The evolved fields are valid at $12 \mathrm{~h}$ forecast time. The contour interval is given by the number above each panel. 
Initial T level 33

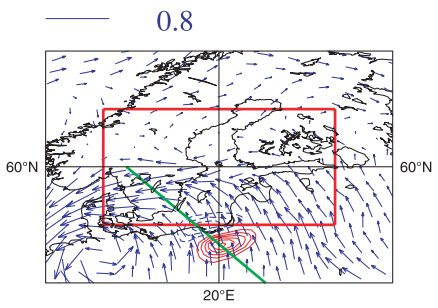

Cross section initial T

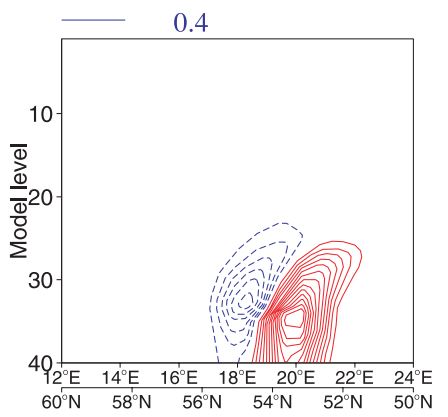

Evolved T level 35

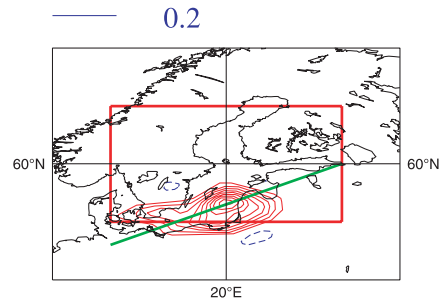

Cross section evolved $\mathrm{T}$

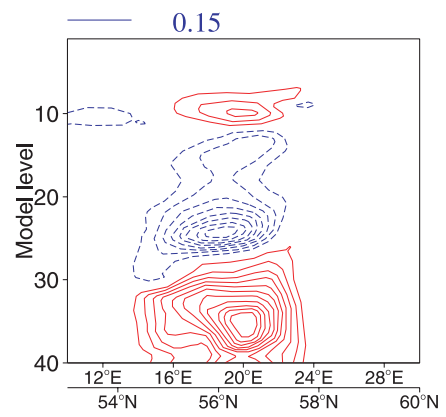

Evolved CAPE

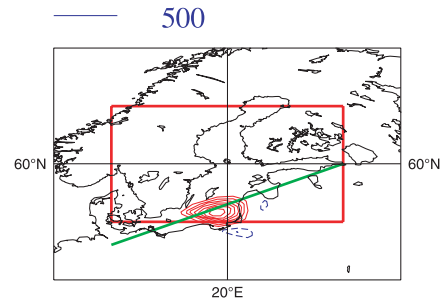

Cross section evolved $q$

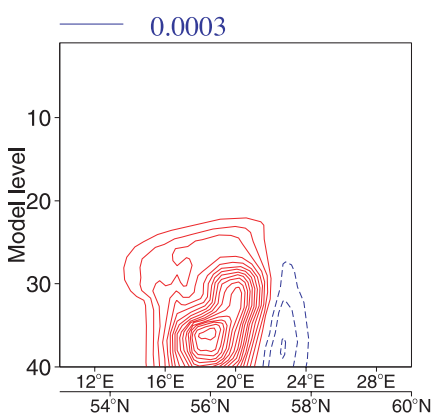

Fig. 4. Leading CAPE-SV for August 21 at 18 UTC, initial temperature and background wind at model level 33 (top left-hand panel) cross section initial temperature (bottom left-hand panel), evolved temperature at level 35 (top middle panel), cross-section temperature (bottom middle panel), Evolved CAPE (top right) and cross-section specific humidity (bottom right-hand panel). The vertical cross sections are along the green line in the upper plot. The red box indicates the target area for the SVs. Positive perturbations (solid red), negative perturbations (dashed blue). The evolved fields are valid at $12 \mathrm{~h}$ forecast time. The contour interval is given by the number above each panel.

Initial T level 33

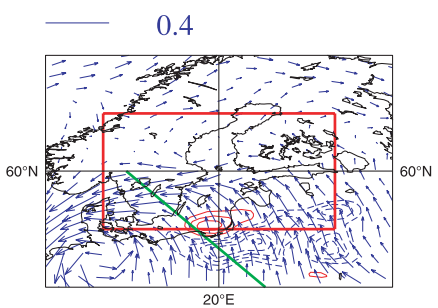

Cross section initial T

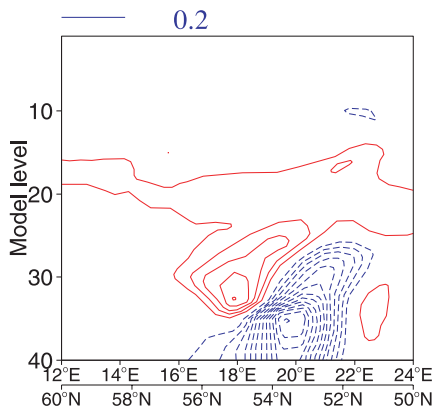

Evolved T level 35

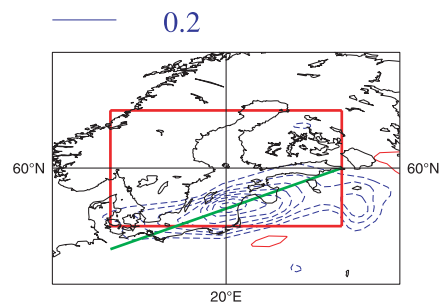

Cross section evolved T

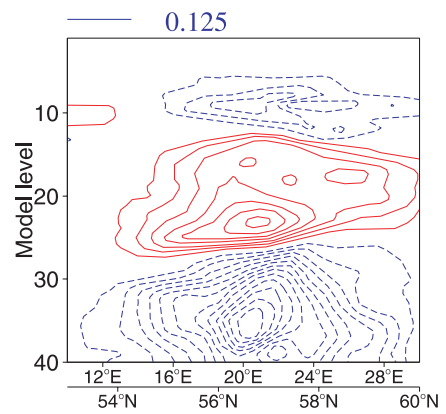

Evolved CAPE

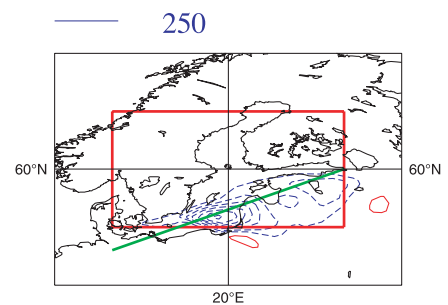

Cross section evolved q

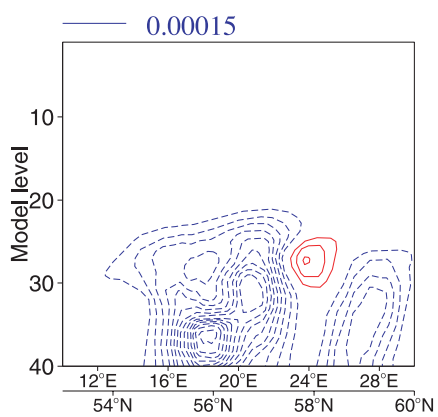

Fig. 5. SCAPE-SVs for August 21 at 18 UTC, initial temperature and background wind at model level 33 (top left-hand panel) cross section initial temperature (bottom left-hand panel), evolved temperature at level 35 (top middle panel), cross-section temperature (bottom middle panel), Evolved CAPE (top right-hand panel) and cross section specific humidity (bottom right-hand panel). The vertical cross sections are along the green line in the upper plot. The red box indicates the target area for the SVs. Positive perturbations (solid red), negative perturbations (dashed blue). The evolved fields are valid at $12 \mathrm{~h}$ forecast time. The contour interval is given by the number above each panel. 
combinations of CAPE-SVs that all have a positive bias in the CAPE field the scale of the CAPE-field of the SCAPE-SVs is larger than the CAPE-field of the CAPE-SVs.

\subsection{Vertical structures}

Figures 6-8 show the average vertical energy distribution inside the projection domain of the TE-SVs, the CAPE-SVs and the SCAPE-SV respectively. For the TE-SVs and the CAPE-SVs the average vertical energy was based on the leading nine SVs. For each day each average field is scaled such that the maximum value is 1 . The upper right-hand plots show the energy distribution inside the projection domain as a function of the analysis time for temperature, kinetic energy and specific humidity in terms of TE. For the TE-SVs on average $80 \%$ of the energy is in the temperature field at initial time. The height where this initial temperature maximum occurs varies between 250 and $450 \mathrm{hPa}$ (model levels 11-17). At evolved time almost all energy is in the kinetic energy (note that the evolved kinetic energy values are divided by 5 in the upper right-hand plot) between 250 and $350 \mathrm{hPa}$ (model levels 11-15). We use a dry total energy norm at initial and final time and so there is no role of the specific humidity field in the SV computation. In the fields that contribute to the total energy the TE-SVs show little variability as a function of time.

For the CAPE-SV on average $70 \%$ of the energy is in the temperature field at initial time with the remaining $30 \%$ in the kinetic energy. These temperature perturbations are always between 850 and $950 \mathrm{hPa}$. For SCAPE-SVs these ratio's are 80 and $20 \%$ for temperature and kinetic energy on average, respectively. There is also much more variability in the height of the initial temperature perturbation. Especially in periods with low singular values the initial temperature field of the SCAPE-SV tends to be higher in the troposphere compared to the CAPE-SVs.

At evolved time the SCAPE-SVs have more energy in the temperature field compared to the CAPE-SV. The energy in this evolved temperature field is relatively constant as a function of time compared to the energy in the evolved specific humidity field and the evolved kinetic energy. Note that wind perturbations at final time have no impact on the CAPE values. These wind perturbations are however an effective way to create moisture perturbations at final time, which is important in creating CAPE. Both CAPE and SCAPE-SVs mainly affect the specific humidity around $850 \mathrm{hPa}$ (model level 30) with little variability in the vertical structure compared to the evolved kinetic energy and temperature.
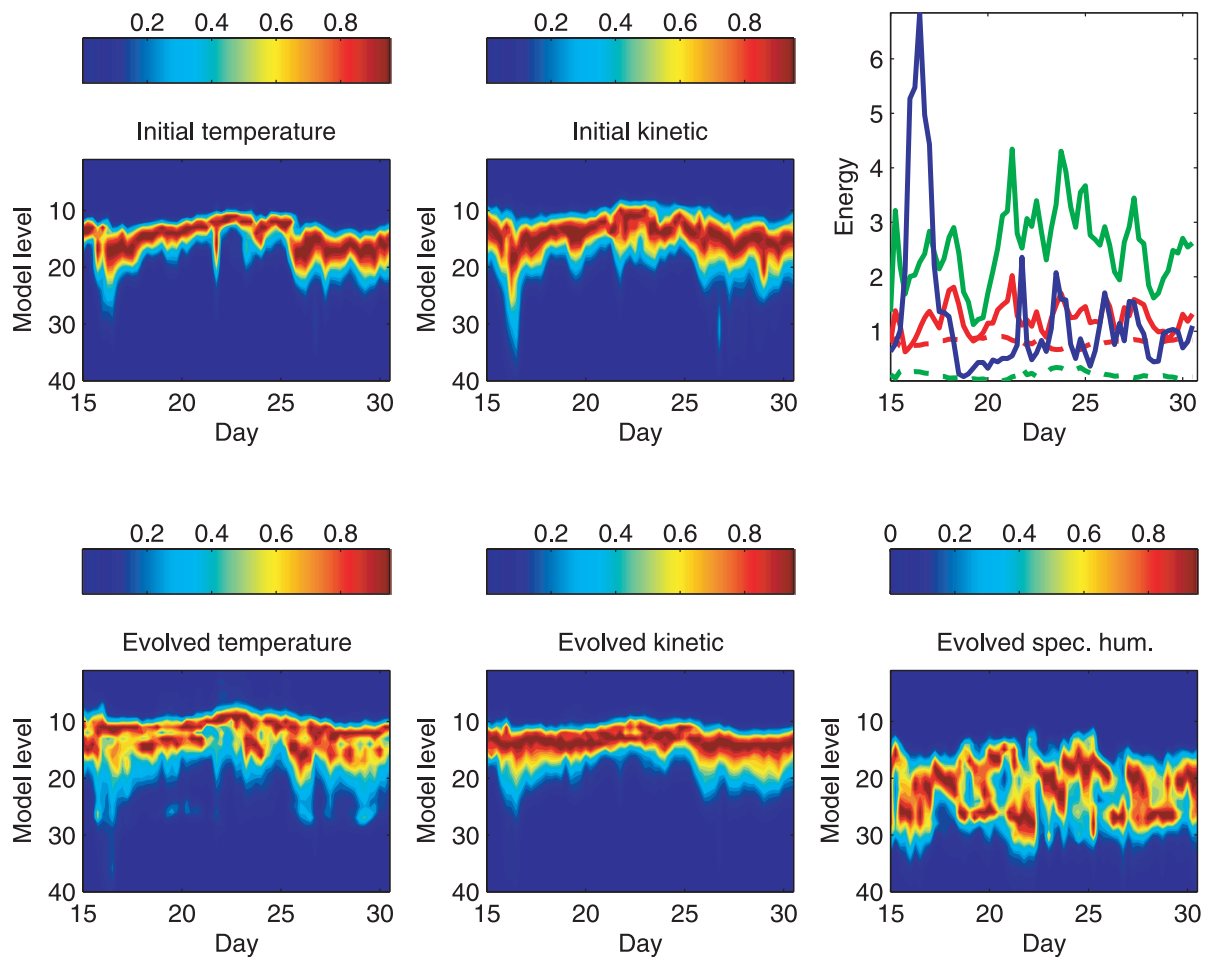

Fig. 6. Scaled average energy distribution of the TE-SV as a function of analysis time. Initial temperature (top left-hand panel) initial kinetic (top middle panel), evolved temperature (bottom left-hand panel), evolved kinetic (bottom middle panel) and evolved specific humidity (bottom right-hand panel). Each field is scaled such that the maximum is 1 . The total energy inside the projection domain for each field is shown in the upper right-hand panel with temperature (red) kinetic energy (green) and specific humidity (blue). Values at initial time (dashed) values at evolved time (solid). The evolved kinetic energy is divided by 5 in the upper right-hand plot. 

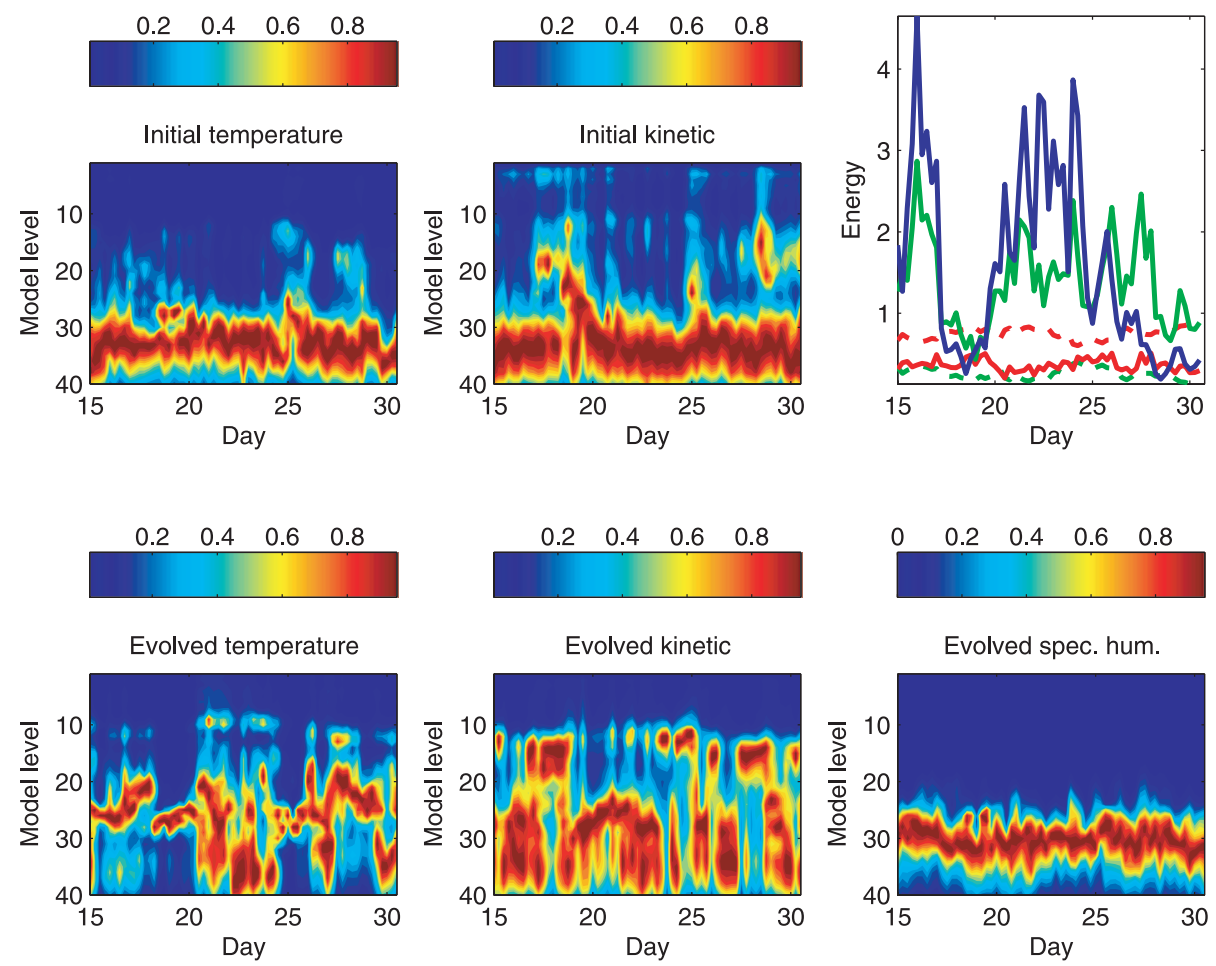

Fig. 7. Scaled average energy distribution of the CAPE-SV as a function of analysis time. See caption Fig. 6. The evolved specific humidity energy is divided by 2 in the upper right-hand plot.
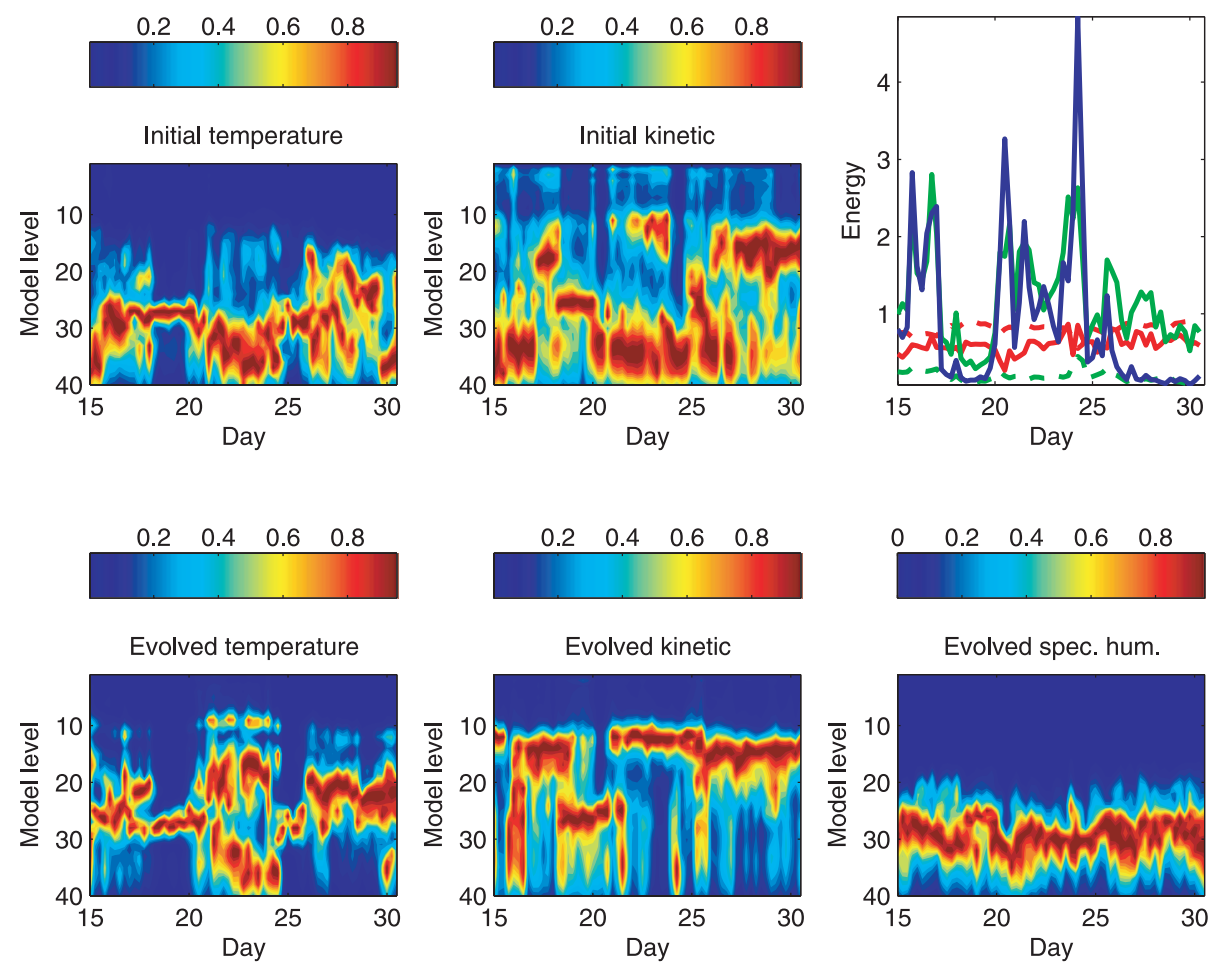

Fig. 8. Scaled energy distribution of the SCAPE-SV as a function of analysis time. See caption Fig. 6. The evolved specific humidity energy is divided by 2 in the upper right-hand plot. 

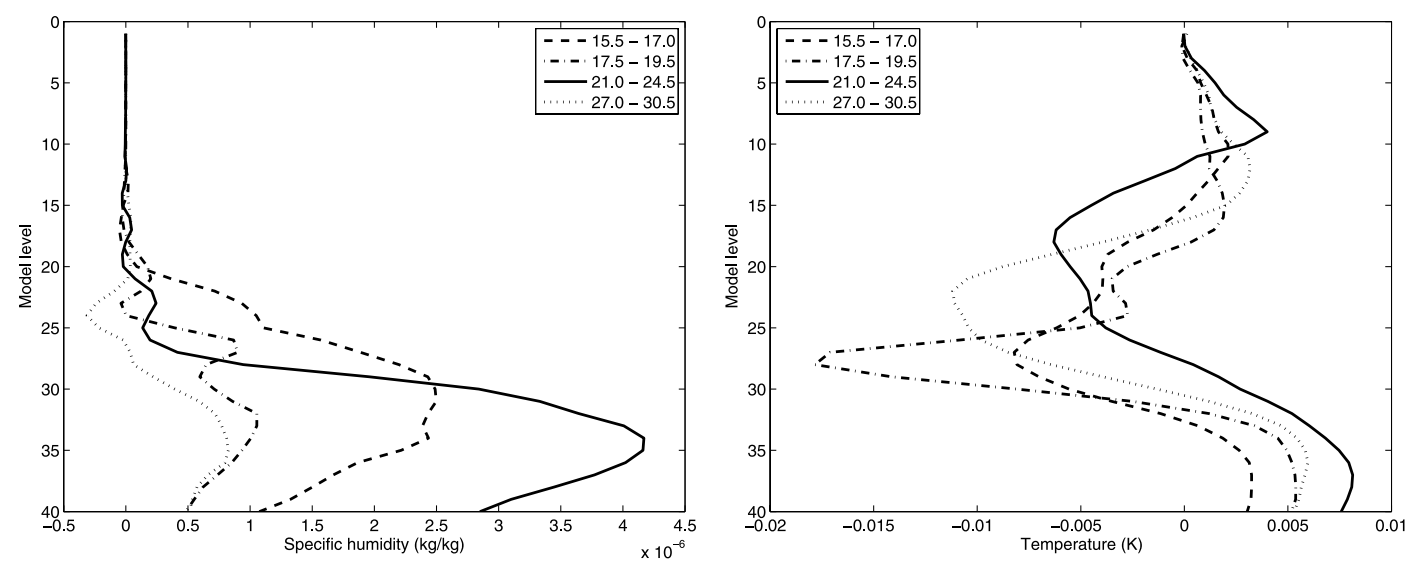

Fig. 9. Horizontally averaged perturbation specific humidity field (left-hand panel) and perturbation temperature field (right-hand panel) inside the projection domain for the SCAPE-SVs averaged over four time periods. Period 1: August 15, 06 UTC to August 17, 00 UTC (dash) period 2: August 17, 06 UTC to August 19, 06 UTC (dash-dotted) period 3: August 21, 00 UTC to August 24, 06 UTC (solid) and period 4: August 27, 00 UTC to August 30, 6 UTC (dotted).

The results presented here all used a dry total energy norm at initial time (no specific humidity perturbations). We performed experiments with SVs with specific humidity perturbations included at initial time using different values for the coefficient in the TE-norm, which measures the energy content in the specific humidity field (see Barkmeijer et al., 2001). What we observed was that part of the energy was in the specific humidity field at initial time, as expected, but the structures of the temperature and wind field were equal to SVs computed without allowing specific humidity perturbations. This is probably related to the exclusion of important diabatic physics in the tangent linear model.

We selected four time periods to examine in more detail the large fluctuations in the evolved specific humidity and the vertical extent of the evolved temperature field for the SCAPE-SVs (see caption Fig. 9 for the definition of the four time periods).

In the first and third period the evolved specific humidity field is dominant in terms of TE. In addition, this third period is characterized by a large vertical extent in the evolved temperature field and high singular values. The second and fourth period are characterized by low values for the evolved specific humidity field. Figure 9 shows the horizontally averaged evolved perturbation of temperature and specific humidity inside the projection domain averaged over the four periods. For all periods we observe that the CAPE-SVs increase the temperature and specific humidity of the parcel, defined as the average condition in the lowest $50 \mathrm{hPa}$ (lowest 5 model levels). This effect is the strongest for the third period with the highest singular values. Furthermore, the specific humidity and lapse rate are increased above the lowest $50 \mathrm{hPa}$. The height over which the lapse rate is increased strongly depends on the synoptic situation. During the second period the lapse rate is increased in a very shallow region around $850 \mathrm{hPa}$ while for the third period the lapse rate over the entire layer from 950 and $400 \mathrm{hPa}$ is increased. This dependence of the vertical temperature profile on the synoptic situation will be difficult to account for in cost functions defined at single model levels or in the planetary boundary layer such as used in Xu et al. (2001). The heating and moistening of the parcel will, in most cases, reduce the convective inhibition and will therefore decrease the lifting needed for a parcel to reach its level of free convection. Therefore, the CAPE-SVs are beneficial to all three necessary ingredients for deep convection mentioned in the introduction.

From Fig. 8 we observe that the evolved specific humidity field of the SCAPE-SVs in terms of TE have the largest impact around $850 \mathrm{hPa}$ (model level 30), while in Fig. 9 the maximum occurs at $950 \mathrm{hPa}$ (model level 35). The effect can be seen most clearly for the third period in Fig. 9. This is caused partly because the model levels represent less mass in the lower troposphere with the result that perturbations at lower levels have less impact on the TE. The same effect is observed in the evolved temperature field. Furthermore at higher model levels there are regions with both positive and negative contributions to the specific humidity field such that the average specific humidity perturbation is smaller while in terms of energy there is a maximum (see Fig. 5).

\section{Linearity test twin experiments}

To test the potential usefulness of the tangent linear model we performed twin experiments for the 'Finnish case' similar to the non-linear test in Vukićević and Errico (1993). A necessary condition for the tangent linear model to be potentially useful is that the error growth in the non-linear run behaves linearly according to a predefined error measure. Figure 10 shows the CAPE perturbation at initial and final time using the leading CAPE-SV as initial condition perturbation. The leading CAPE-SV was multiplied by 0.1 resulting in a perturbation with maximum values for temperature of $0.58 \mathrm{~K}$ and for wind of $0.38 \mathrm{~m} \mathrm{~s}^{-1}$. As we 

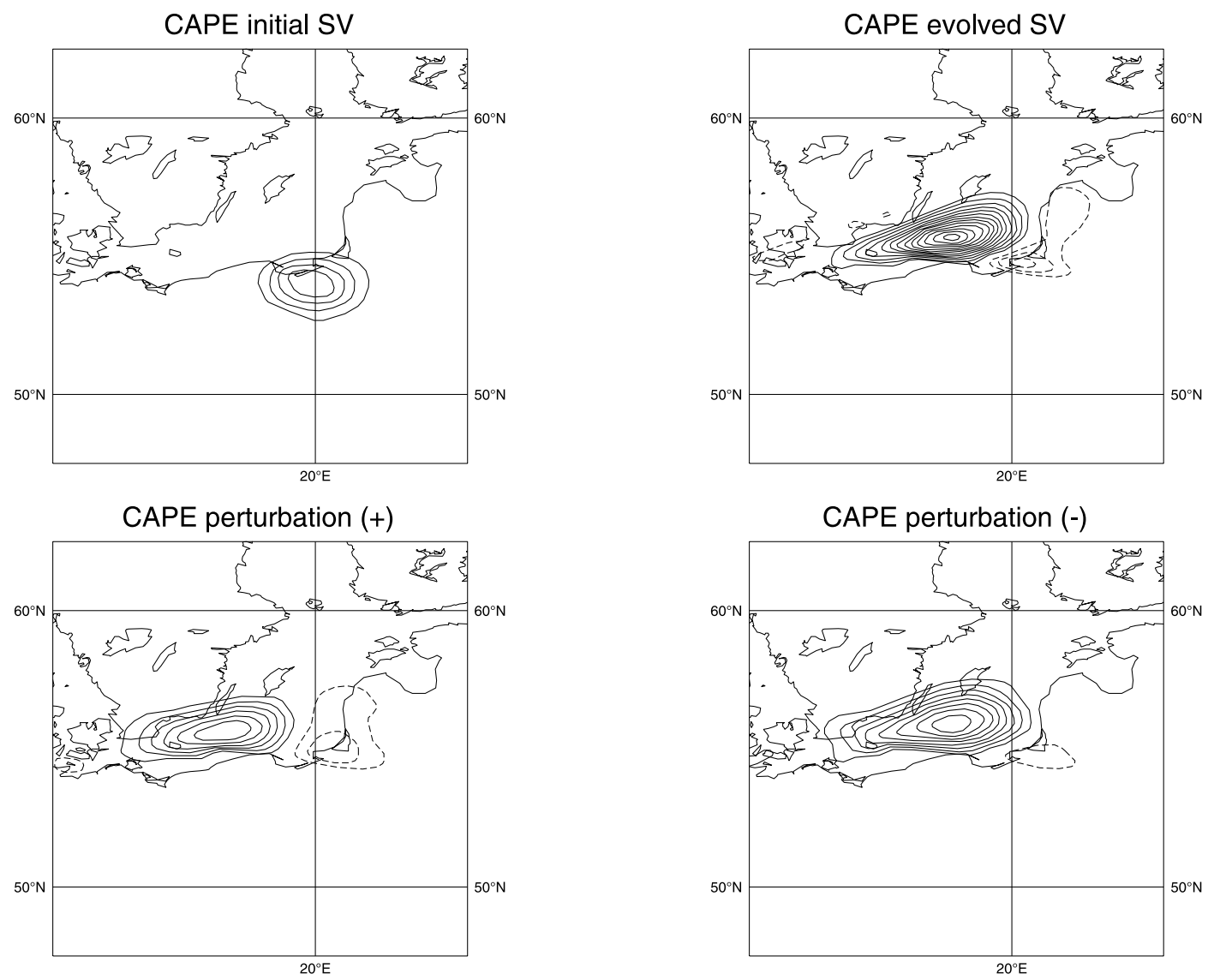

Fig. 10. CAPE perturbations at initial time (top left-hand panel) and final time in the tangent linear model (top right-hand panel) and in the two non-linear runs (bottom left- and bottom right-hand panels). The CAPE values in the (-) run are multiplied with -1 for ease of comparison. The contour interval is $30 \mathrm{~J} \mathrm{~kg}^{-1}$ in all panels.

used a dry TE-norm to constrain the initial perturbations, the CAPE perturbation over the north of Poland at initial time is determined completely by the temperature field. At final time the specific humidity is the dominant field in terms of energy and the CAPE perturbation is now located over the Baltic sea. As shown in Fig. 10 the result of adding or subtracting the CAPE$\mathrm{SV}$ gives very similar CAPE perturbations in the non-linear runs indicating that the tangent linear assumption is valid if CAPE is used as verification measure. The tangent linear model however overestimates the amplitude of the CAPE perturbation.

Although these results look promising we noticed that already after $1 \mathrm{~h}$ small scale noise with low amplitude is present in the boundary layer for the entire integration domain in the twin experiment runs for the temperature and wind field. We noticed that vertical integrals such as CAPE are rather insensitive to this small-scale noise for reasonably sized initial perturbations, but similarity indices between the full three-dimensional fields are low. When the amplitude of the initial condition perturbation is decreased the noise starts to interfere too much with the SV growth when twin experiments are performed leading to lower similarity indices. Similar behaviour has been observed by Vukićević and Errico (1993). They conclude that the dominant mechanism for very small perturbation evolution is the forcing of gravity waves for the regions and periods where the convection is sensitive to $2 \Delta t$ oscillations. We will investigate the loss of linearity of HIRLAM for these cases in a follow-up paper. Note that the loss of linearity for the small amplitude twin experiment does not imply that the results of the tangent linear model presented here are invalid but might actually reveal problems with the non-linear model itself.

\section{Conclusions and discussion}

The feasibility of using the singular vector technique to create initial condition perturbations for short range ensemble predictions has been investigated. For this a new final time norm based on CAPE has been introduced. We have studied the properties of two types of CAPE-SVs and compared their structure with TESVs for a 2-week summer period in 2007 during which a severe mesoscale convective system in the south west of Finland was observed. Both types of CAPE-SVs show a clear increase in the singular values indicating that small perturbations can give rise 
to large CAPE perturbations during the mesoscale convective event. During this period there is a daily pattern in the singular values with lower values for singular vectors starting from the 00 and 18 UTC analysis. TE-SVs show no increase in the singular values for this period and there is no daily pattern.

During the 2-week period all SCAPE-SVs increase CAPE at final time by increasing the specific humidity and temperature of the parcel and at the same time increasing the lapse rate above the parcel. The increase in moisture content and temperature in the low troposphere will also decrease the absolute value of $\mathrm{CIN}$ and therefore reduce the lift needed for parcels to reach their LFC. So, although the CAPE-SVs do not provide the lift needed to reach the LFC, most convection schemes will be easier triggered in runs that are perturbed with CAPE-SVs. Therefore adding CAPE-SVs to the initial condition is beneficial to all three necessary ingredients for development of deep convection mentioned in the introduction (Doswell, 1987). Another useful property of both types of CAPE-SVs for short range EPS is that their activity mainly takes place in the lower troposphere. This in contrast to TE-SVs with OT $=12 \mathrm{~h}$ which predominantly remain in the high troposphere (above $500 \mathrm{hPa}$ ). Note that although the specific humidity component has the largest contribution in terms of TE in the evolved CAPE-SVs there is also perturbation growth in the kinetic energy component. We plan to explore the impact of this different behaviour in the context of a shortrange ensemble systems (GLAMEPS, Iversen et al., 2007) focussing particularly on the performance with respect to weather parameters.

For certain vertical temperature and humidity profiles parcels will never reach their LFC. In those case there are no infinitesimal perturbations that will produce CAPE and therefore the CAPE operator acts as a projection operator for these points. This also happens for cost functions based on precipitation (Mahfouf and Bilodeau, 2007) where only levels where supersaturation is diagnosed in the background state contribute to the norm. One solution to avoid this projection is to change the lower integration limit in the CAPE integral to the initial parcel height instead of the LFC. This amounts to subtracting the energy needed to reach the LFC (the convective inhibition) from the CAPE. Optimal perturbation based on this norm will decrease the absolute value of CIN for those specific humidity and temperature profiles with zero CAPE and both decrease the absolute value of CIN and increase CAPE for profiles with positive CAPE values.

We have shown that the CAPE-norm can be used in the method of $\mathrm{Xu}$ et al. (2001) to create optimal perturbations with a cost function that measures the mean CAPE inside a projection domain. Short range (ensemble) forecasting has to deal with many forecast aspects. Several indices have been developed to evaluate the convective and severe weather potential of the atmosphere such as the Showalter index, lifted index, total totals, total energy index, severe weather threat, bulk Richardson number, helicity index, vorticity generation potential, etc. (Blanchard, 1998; Peppler, 1988). For indices that depend differentiably on the model state the same method can be used to derive a norm and create optimal perturbations. These optimal perturbations are an interesting alternative for short range EPS based on singular vectors. First of all, each ensemble member can be constructed to focus on a particular forecast aspect by using a norm derived from a related weather index. Note that we can easily adjust the optimization time and the resolution in the adjoint integration, for example, we can choose short optimization times and higher resolutions when norms are used that focus on mesoscale aspects of the forecasts. Secondly, when several indices are being used to compute the optimal perturbations, the impact of each optimal perturbation can be judged on their impact on ensemble skill scores. This information could be used to gradually improve the norms used to create the initial condition perturbations.

\section{Acknowledgments}

We would like to thank Nils Gustafsson, Toon Moene, Ole Vignes and Xiaohua Yang for support during the implementation of the singular vector code in HIRLAM. We thank Theo Opsteegh, Sander Tijm and Wim Verkley for carefully reading earlier versions of the manuscript and two anonymous reviewers for their useful comments.

\section{References}

Barkmeijer, J., Buizza, R., Palmer, T. N., Puri, K. and Mahfouf, J.-F. 2001. Tropical singular vectors computed with linearized diabatic physics. Q. J. Roy. Meteor. Soc. 127, 685-708.

Blanchard, D. O. 1998. Assessing the vertical distribution of convective available potential energy. Weather Forecast. 13, 870-877.

Doswell, C. A. III 1987. The distinction between large-scale and mesoscale contribution to severe convection: a case study example. Weather Forecast. 2, 3-16.

Doswell, C. A. III and Rasmussen, E. N. 1994. The effect of neglecting the virtual temperature correction on CAPE calculations. Weather Forecast. 9, 625-629.

$\mathrm{Du}$, J. et al. 2009. NCEP short-range ensemble forecast (SREF) system upgrade in 2009. Preprints, 19th Conf. on Numerical Weather Prediction and 23rd Conf. on Weather Analysis and Forecasting, Omaha, Nebraska, Amer. Meteor. Soc. Weather and Forecasting paper 4A.4.

Errico, R. M., Raeder, K. and Ehrendorfer, M. 2004. Singular vectors for moisture-measuring norms. Q. J. Roy. Meteor. Soc. 130, 963-988.

Errico, R. M., Raeder, K. D. and Fillion, L. 2003. Examination of the sensitivity of forecast precipitation rates to possible perturbations of initial conditions. Tellus 55A, 88-105.

Fillion, L. and Bélair, S. 2004. Tangent linear aspects of the Kain-Fritsch moist convective parameterization scheme. Mon. Weather Rev. 132, 2477-2494.

Hágel, E. and Horányi, A. 2007. The ARPEGE/ALADIN limited area ensemble prediction system: the impact of global targeted singular vectors. Meteorol. Z. 16, 653-663.

Hohenegger, C. and Schär, C. 2007. Atmospheric predictability at synoptic versus cloud-resolving scales. Bull. Am. Meteorol. Soc. 88, 1783-1793. 
Homar, V., Stensrud, D. J., Levit, J. J. and Bright, D. R. 2006. Value of human-generated perturbations in short-range ensemble forecasts of severe weather. Weather Forecast. 21, 347-363.

Iversen, T., Frogner, I.-L., Sattler, K., Ivatek-Sahdan, S., Barkmeijer, J. and co-authors. 2007. Glameps-the Hirlam/Aladin grand limited area model ensemble prediction system. ECMWF Workshop on Ensemble Prediction, 7-9 November 2007, 95-106, ECMWF.

Janisková, M. 2003. Physical processes in adjoint models: potential pitfalls and benefits. In Proceedings of the ECMWF Seminar on Recent Developments in Data Assimilation for Atmosphere and Ocean, 179-192, Reading, UK, ECMWF.

Janisková, M., Thépaut, J. N. and Geleyn, J. F. 1999. Simplified and regular physical parameterizations for incremental four-dimensional variational assimilation. Mon. Weather Rev. 127, 26-45.

Johns, R. H. and Doswell, C. A. III 1992. Severe local storms forecasting. Weather Forecast. 7, 588-612.

Leutbecher, M. and Palmer, T. N. 2008. Ensemble forecasting. J. Comput. Phys. 227, 3515-3539.

Mahfouf, J.-F. and Bilodeau, B. 2007. Adjoint sensitivity of surface precipitation to initial conditions. Mon. Weather Rev. 135, 2879-2896.
Palmer, T. N., Gelaro, R., Barkmeijer, J. and Buizza, R. 1998. Singular vectors, metrics, and adaptive observations. J. Atmos. Sci. 55, 633-653.

Peppler, R. A. 1988. A review of static stability indices and related thermodynamic parameters. Illinois State Water Survey Misc. Publ 104.

Schultz, D. M. and Schumacher, P. N. 1999. The use and misuse of conditional symmetric instability. Mon. Weather Rev. 127, 2709-2732.

Stensrud, D. J., Bao, J. W. and Warner, T. T. 2000. Using initial condition and model physics perturbations in short-range ensemble simulations of mesoscale convective systems. Mon. Weather Rev. 128, 2077-2107.

Undén, P., et al. 2002. HIRLAM-5 scientific documentation. SMHI Norrköping, Sweden.

Vukićević, T. and Errico, R. M. 1993. Linearization and adjoint of parameterized moist diabatic processes. Tellus 45A, 493-510.

Xu, M., Stensrud, D. J., Bao, J. W. and Warner, T.T. 2001. Applications of the adjoint technique to short-range ensemble forecasting of mesoscale convective systems. Mon. Weather Rev. 129, 1395-1418.

Yang, X. 2005. Analysis blending using spatial filter in grid-point model coupling. Hirlam Newslett. 48, 49-55. 